\title{
हिमाचल प्रदेश में रागध्यान चित्रावलियों के विभिन्न क्षेत्रों की लोक शैलियां व उनके प्रयुक्त रंग रेखाएं
}

मीनाक्षी

हिमाचल प्रदेश में रागध्यान चित्रावलियों की विभिन्न शैलियाँ हिमाचल प्रदेश की चित्रकला भारतीय चित्रकला के इतिहास में विशिष्ट स्थान रखती है। विश्व में हिमाचल प्रदेश की चित्रकला ख्याति प्राप्त कर चुकी है। चित्रकला के उदाहरण लघुचित्रों कागज़ों पर बनाए गए थे और भिति चित्र मन्दिरों, राजमहलों, बावड़ियों तथा निजी भवनों की दीवारों और छतों पर उन्हे सुशेभित करने के लिए अंकित किए गए थे। चित्रकला का जन्म हजारों वर्ष पूर्व हो गया था इसका प्रमाण आदि मानव द्वारा निर्मित गुफा चित्र हैं। हिमाचल प्रदेश की चित्रकला हमारी सभ्यता व संस्कृति की अमूल्य धरोहर है।

हिमाचल प्रदेश की चित्रकला की सुन्दर कृतियाँ 19 वीं और 20 वीं शताब्दी में प्रकाश में आई। 10 वीं शताब्दी में मैट्काफ ने कांगड़ा में इस शैली के कुछ चित्रों की खोज की। 20वीं शताब्दी में डॉ. आनन्द कुमार स्वामी ने 1908-10 ई. स्वामी ने राजपूत कला को मुगल कला से भिन्न बताया। उन्होंने राजपूत कला को दो भागों पहाड़ी कला तथा राजसथानी कला में विभक्त किया। पहाड़ी कला का क्षेत्र पंजाब की पहाड़ी रियासतें (वर्तमान हिमाचल प्रदेश) तथा राजस्थानी कला का क्षेत्र राजस्थान का मैदानी क्षेत्र था। 1916 ई. में डां. आनन्द कुमार स्वामी की पुस्तक "राजपूत पेटिंस" में पहाड़ी कलाकृतियों की सांस्कृतिक भूमिका पर प्रकाश डाला।

1931 ई. में जे.सी. फ्रैंच की पुस्तक हिमालयन आर्ट प्रकाशित हुई। यह पुस्तक उन्होंने 1930 ई. में गुलेर, मण्डी, कुल्लू, अर्की, चम्बा, तथा लम्बागांव आदि रियासतों का दौरा करने के पश्चात् लिखी। 1952 ई0 में डब्ल्यू.जी. आर्चर की दो पुस्तकें, "इण्यिन पेंटिग्स इन द पंजाब हिल्ज" और "कांगडा पेटिंगस" प्रकाशित हुई। पहली पुस्तक में उन्होने विभिन्न शैलियों जो उस समय हिमाचल प्रदेश में प्रचालित थी, का विश्लेषणात्मक अध्ययन किया। आर्चर के अध्ययन से प्रेरित होकर डा. एम.एस. रूधावा कांगड़ा घाटी गए और उन्होने इस विषय पर गहन अध्ययन किया तथा इससे सम्बध्धित कई निबन्ध व पुस्तक लिखी। इनकी पुस्तकें बसोहली पेंटिग्स "कांगड़ा वैली पेंटिग्स" तथा कृष्णा लिंजेण्ड़ इन पहाड़ी पेंटिग्स अपना महत्वपूर्ण स्थान रखती है। कार्ल खण्डेलवाला की बृहत पुस्तक 'पहाड़ी मिनीएचर पेंटिगस' में बहुत खोजपूर्ण सामग्री उपलब्ध है। इसके अलावा डा. मुल्क राज आनन्द और डॉ. बी. एन. गोस्वामी आदि विद्वानों ने भी पहाड़ी चित्रकला का अध्ययन किया।

\section{हिमाचल प्रदेश विभिन्न शैलियां}

हिमाचल प्रदेश की चित्रकला का जन्म कब और कहा हुए इसके सन्दर्भ में विद्वानों में मतभेद है कुछ विद्वानों के अनुसार इस चित्रकला का जन्म जन्मू के पहाड़ी क्षेत्र बसोहली में हुआ इनके अनुसार सर्वप्रथम मुगल दरबार से निष्कासित कलाकारों का दल बसोहली पहुंचा और उन्होंने वहां की लोककला से प्रेरित होकर बसोहली शैली का निर्माण किया। इसके बाद इसका विकास अन्य पहाड़ी रियासतों में हुआ।

अन्य विद्वानों के अनुसार पहाड़ी कला का जन्म गुलेर से हुआ। 1780 ई0 में जब गुलेर शैली अपने पूर्ण निखार में थी तो उसने कांगड़ा में प्रवेश किया और कांगड़ा शैली के नाम से प्रसिद्ध हुई।

\section{कांगड़ा शैली}

कांगड़ा शैली का जन्म गुलेर में हुआ था। गुलेर के राजाओं के सम्बंध मुगल सम्राटों-शाहजहाँ जहांगीर और औरंगजेब से अच्छे थे। इसलिए यह सम्भव है कि गुलेर गुलेर के कुछ कलाकार दिल्ली गए हो या दिल्ली से मुगल कलाकार गुलेर आए हो। यह भी सम्भव है कि औरंगजेब द्वारा निष्कासित कलाकारों ने गुलेर में आकर शरण ली हो। औरंगजेब ने जब अपने राज्य से कलाकार

1 पी.एच.-डी. शोध-छात्रा, इन्दिरा संगीत कला विश्वविद्यालय, खैरागढ़। 
भगाए तो उन्होने इधर-उधर आश्रय की खोज की। इसी समय वे गुलेर भी पहुंचे होगे। अन्य पहाड़ी राज्यों से गुलेर सब से नजदीक था।

चित्रकला का जन्म गुलेर में ही नही हुआ। वास्तव में हिमाचल प्रदेश में इसका जन्म लोककला के रूप में हुआ। हिमचल प्रदेश विभिन्न रियासतों में बंटा हुआ था। इनमें प्राचीन काल से ही लोक कला किसी न किसी रूप में विद्यमान थी। लोक कला के इस प्रचलित रूप को राजाओं की धार्मिक प्रवृति अथवा उनके कला प्रेम के कारण राजाश्रय प्राप्त हुआ तो इसमें निखार आता गया। यह समय के साथ सुसंस्कृत होती हुई पहाडी कलम या कांगडा की कलम के नाम से प्रसिद्ध हुई।

\section{कांगड़ा कलम}

पहाड़ी चित्रकला कांगड़ा कलम के नाम से जानी गई है। कांगड़ा कलम को ही पहाड़ी कला का पर्यायवाची समझा जाता है। सम्पूर्ण पहाड़ी कला के इतिहास में कांगड़ा कलम एक सर्वोच्च उपलब्धि रही और अन्य क्षेत्रों में जहाँ भी पहाड़ी कला का विस्तार देखने में आता है। किन्ही भी कांगड़ा सी उपलब्धि रंग और रेखाओं की अत्यन्त सुरूचिपूर्ण भावभीनी झलकिया देखने में नहीं आती। कला आन्दोलन में जो विशिष्टतम रहा वह कांगड़ा कलम है। पहाड़ी कला की यह उपलब्धि कांगड़ा के शासक महाराज संसार चन्द (1775-1823) के राज्यकाल की देन थी।

राजा संसर चन्द का कला-प्रेमी होना पहाड़ी चित्रकला के इतिहास की महत्वपूर्ण घटना थी। उनका निजी कला प्रेम ही पहाड़ी चित्रकला को विशिष्ट स्थान देने में समर्थ रहा। पड़ोसी राज्यों विशेषतः गुलेर से कलाकारों का राजा संसारचन्द के पास आना एक स्वाभावित प्रक्रिया बन चुकी थी और उनके आश्रय में हर कलाकार अपनी प्रतिमा को उभारने और अपनी कला को निखारने का सुअवसर प्राप्त करता रहा। उनकी कला प्रियता का अनुमान इस बात से भी लगता है कि भारत ही क्या बाहर के संग्रहलायों में भी राजा संसार चन्द के शासन काल के चित्र देखने से आते है। प्रेम को ही क्यों सबसे अधिक और समर्थ अभिव्यक्ति मिली, उसके लिए स्वयं राजा संसार चन्द का निजी प्रणययुक्त जीवन तथा दृश्टिकोण उत्तरदायी है।

कांगड़ा कलम की नोक ने जिस भी विषय को छुआ वही उसकी उपलब्धि बन गई। जिसकी समता न तो उससे पूर्व की कला में मिलती है और न ही उसकी समयकालीन कला में चाहे रेखाओं का प्रवाह है अथवा रंगों का आयोजन लयात्मकता है अथवा सन्तुलन, आकृति-अंकन है अथवा वास्तु और प्रकृति का चित्रण, इन सभी में कांगड़ा कलम की अपने देन है। उसकी अपनी विशिष्टता है जिसमें हमें ढूढ़ने पर भी कोई दोष अथवा त्रुटि नहीं मिलती। चित्र अपनी सम्पूर्णता से मन को खींचता है, उसे रस-विभोर करता है।

कांगड़ा कला ने कांगड़ा कलम के रूप में अपनी सबसे सुन्दर कलात्मक कृतियां भेंट की थी। लेकिन महाराजा संसार चन्द की मृत्यु के बाद इसके भरणपोषण की स्थिति का अन्त हो गया। रास्तों के खुले जाने पर बाहरी दुनिया से सम्पर्क बन गया था। कलाकारों के लिए कला की अनुशीलता के अनुकूल परिस्थितियां न रही थी। कलाकार एक प्रकार से विस्थापित अनुभव कर रहे थे। वे कामकाज के अन्य धन्धे ढूंढने लगे थे। कांगड़ा का भूचाल जो 4 अप्रैल, 1905 के दिन आया उसने निश्चिय रूप से कांगड़ा कला का अन्त कर डाला। इस विध्वंस में गिनती की ही कलाकृतियाँ बच सकीं। यही दैवी घटना पहाड़ी कला के विलोप के लिए सबसे अधिक उत्तरदायी रही।

\section{गुलेर कलम}

1780 के आसपास गुलेर शैली अपने सर्वोच्च निखार में नजर आती है। गुलेर कांगड़ा के दक्षिण में स्थित मैदानों के अधिक निकट था। इस कारण इस पर पंजाब का प्रभाव अधिक रहा इसे मुगल संरक्षण भी प्राप्त होता रहा जिससे अन्य पहाड़ी रियासतों में इसकी सैनिक प्रतिष्ठा बनी। राजा रूपचन्द (1610-1635) को मुगल बादशाह शाहजहां का सर्मथन प्राप्त होने के कारण उसने पड़ौसी राज्यों से बाईस लड़ाईयां लड़ीं। रूपचन्द के बाद मानसिंह (1635-61) राजा बना। राजा मानसिंह के भी मुगल साम्राज्य के साथ मित्रतापूर्ण संबंध रहे। 
गुलेर के शासकों में राजा गोबर्धन सिंह चित्रकला के असाधारण पोषक थे। गुलेर के राजा गोबर्धन सिंह की 1773 में मृत्यु हो गई। चम्बा में राजा सिंह (1773-94) का नौ वर्ष की आयु में राज्याभिषेक हुआ। 1775 में उसने जम्मू की सेना को परास्त किया था और 1785 में बसोहली पर विजय प्राप्त की। उनकी कलारूचि को ख्याति मिलने पर अनेक कलाकार उनके यहाँ एकत्रित हुए। यहां उनसे दरबार में अनेक चित्रकारों ने संरक्षण प्राप्त किया। उनके दरबार में कलाकारों की कृतियाँ की परख हो तो थी। कलाकारों को उत्साहित किया जाता था और उनकी उपलक्षियों पर उन्हें पुरस्कार मिलते रहते थे। इस प्रकार राजा संसार चन्द का दरबार कलाकारों के लिए एक बहुत बड़ा आकर्षण और आश्रय रहा।

\section{चम्बा कलम}

पहाड़ी चित्रकला में बसोहली कलम प्राचीनत है। कांगड़ा कलम में हम मुगलकालीन प्रभाव देखे जा सकते है। लेकिन बसोहली कलम ऐसे प्रभाव से मुक्त रही। कांगड़ा कलम अपने सर्वोच्च निखार पर आकर विलुप्त हो गई। लेकिन बसोहली शैली की जड़े जन-जीवन में गहरी चली गई। यही कारण है कि अनेक तत्कालीन पहाड़ी रियासतों में कांगड़ा कलम का उतना प्रभाव न रहा जितना बसोहली कलम का बसोहली प्रभाव को ग्रहण करने में चम्बा का नाम विशेष है। उत्तरोत्तर विकास में चम्बा शैली स्वतन्त्र रूप से परिपक्वता ग्रहण करती गई। आरम्भ में चम्बा राजाओं के जो चित्र बसोहली शैली में प्राप्त हुए है उनमें चम्बा पर बसोहली प्रभाव स्पष्ट होता है। चम्बा स्थित भूरि सिंह म्यूजियम में ही कल्कि और परशुराम विषयक दो चित्र संकलित है। जो रंग और रेखाओं की दृष्टि से बसोहली शैली के नज़दीक है। चम्बा चित्रशेली का प्रारम्भ राजा उदय सिंह (1690-1720) के शासन काल में हुआ। इससे पूर्व भी राजा पृथ्वी सिंह (1641-64) और राजा चतर सिंह (1664-90) के एक एक चित्र उक्त राजाओं के समय में न बनकर बाद में बने हों। इन्हें चम्बा के प्राचीनतम् चित्र माना जाता है।

चम्बा कलम का उदय 17 वीं शताब्दी का मध्य माना जाता है। पड़ोसी राज्य बसोहली और गुलेर में शासन व्यवस्था डांवाडोल होने लगी तो चितेरे राज्य-प्रक्षय की खोज में चम्बा बस गए। 18 वीं शताब्दी के अंतिम चरण में चम्बा की अपनी निजी शैली स्थापित हुई। चम्बा शैली पर बसोहली और गुलेर का प्रभाव देखा जा सकता है।

\section{मण्डी कलम}

मण्डी कलम के उदय, विकास और उपलब्धियों से यह स्पष्ट है कि यह राज्य प्रश्रय में पनपी। कलाकार लोक-कला के प्रति सदा ऋणी रहा। उसने ऐसे विषयों को हुआ जो लोक जीवन की अन्तरंग झांकियां प्रस्तुत करते हैं। शिल्प की दृष्टि से मण्डी कलम को तीन भागों में बांटा जा सकता है

1. 1804 तक लोक शेली का प्रभाव

2. 1804 से 1846 तक पड़ौसी शैलियों का प्रभाव

3. 1846 से 1912 तक पाश्चात प्रभाव

सन् 1750 तक मण्डी कलम में निखार आ गया था। इसी काल में बना राजा शमशेर सेन के विवाह का चित्र-शिल्प, आकृति-अंकन तथा संयोजन की दृष्टि से काफी परिष्कृत ही चित्र से यह आभास होता है कि इस काल में मण्डी कलम से सौभ्यता आ गई थी। चित्र की आत्मा लोक कला के अनुरूप ही थी पर उससे सरंचना लालितपूर्ण हो गई।

\section{बिलासपुर कलम}

बिलासपुर कलम का इतिहास राजा दीपचन्द (1650-67) से आरंभ्भ होता हौ राजा दीपचन्द के समय भारत में औरंगजेब का राज्य था। औरंगजेब को ललित कलाओं से चिढ़ थी। दिल्ली दरबार के शाहजहां कालीन चितेरे 17 वीं शताब्दी के उतरार्द्ध में बिखरने आरम्भ हुए और अन्य पहाड़ी रियासतों की भांति बिलासपुर में भी पहुंचे। इन चितेरों ने अपने पहाड़ी आश्रयदाताओं का जिस ढंग से चित्रण किया है उससे मुगल कलम से प्रभावित होने के संकेत मिलते है। 
बिलासपुर के राजा दीपचन्द (1741-78) में राज्यकाल में रियासत ने काफी उन्नति की थी। राजा के कलाप्रिय शासक होने के कारण अपने यहाँ कई कलाकारों को प्रश्रय दिया। इस समय गुलेर तथा बसोहली के चित्रकार बिलासपुर में बसने आरम्भ हो गए थे। इस काल में बने चित्रों में बसोहली तथा गुलेर कलमों की छाप है।

\section{हिमाचल प्रदेश की रागध्यान चित्रावलियों (रागमालाओं) में प्रयुक्त रंग रेखाएं एवम प्रतीक}

रंग-रेखाओं के सन्तुलित और प्रभावपूर्ण प्रयोग के कारण ही भारतवर्ष की पारम्परिक चित्रकला ने विश्वभर में ख्याति अर्जित की है। सभी पारम्परिक चित्र शैलियाँ में चाहे वह पहाड़ी शैली हो या राजपूत शैली, मुगल शैली हो अथवा यर्थाथवादी शैली सभी का रंग-रेखाओं के सन्तुलित आधारपर ही अंकन हुआ है। चित्रित पात्रों का ओज और माधुर्य सभी को रंग रेखाओं का उत्तम प्रयोग समृद्ध बनाता है। रंग और रेखाओ का प्रयोग जितना संगीतपूर्ण, समीचीन और सन्तुलित होगा, दर्शक के मानस पटल पर उसका प्रभाव उतना ही स्थायी होगा। प्रत्येक रंग का अपना विशेष गुण होता है प्रत्येक रेखा का अपना एक कथ्य होता है।

\section{मूल रंग}

पारम्परिक भारतीय विचारधारा के अनुसार विभिन्न रंगों के भिन्न-भिन्न गुण माने गए हैं जो इस प्रकार से हैं-

लाल रंग अनुराग तथा क्रोध का प्रतीक माना गया है। इस रंग को देखकर मनुष्य तो क्या पशु तक भड़क उठते है। खून का रंग लाल होता है इसलिए मनोवैज्ञानिक रूप से वह रंग, युद्ध अथवा खतरे का द्योतक हैं। ग्रहों में भी मंगल का रंग लाल बताया गया है देखने में भी मंगल ग्रह लाल दिखाई पड़ता है। सूर्य का रंग भी लाल ही माना गया है। अतः वह तेज, उद्दीपन या उत्तेजना का प्रतीक है। चित्रों में वीभत्स क्रोध, घृणा, पाप आदि भावों की सृष्टि इसी रंग के आधार पर की जाती है।

पीला रंग प्रसन्नता और उत्साह का द्योतक है। यह सम्भवतया इसलिए माना गया है कि कड़ाके की सर्दी बीत जाने के बाद जब बसन्त का आगमन होता है तो मन में उत्साह और हर्ष भर जाता है।

यही रंग बसन्त का होता है। जब नई फसल पकती है तथा चारों और सरसों के पीले फूल खिल उठते हैं। वृहस्पति का रंग भी पीला माना है शान्ति, सदभावना, उत्साह, उमग और उल्लास के वातावरण की सृष्टि हेतु पीले रंग को आधार बनया गया है।

नीला रंग महानता और गम्भीरता का प्रतीक माना गया है। वह कदाचित् इसलिए कि पानी का रंग भी नीला होता है समुद्र और आसमान में जो विशालता का भाव है वी नीले रंग के साथ जुड़ा हुआ है इसलिए चित्र में भी उक्त भावों की सृष्टि नीलवर्ण के रंगों द्वारा ही की जाती है।

हरे रंग में मूलतः नीला रंग और पीला रंग मिले होते है नीला रंग पानी का और पीला रंग मिट्टी का होता है। प्रकृति का भी यही रंग है।

सफेद रंग जो की शांति और समृद्धि का द्योतक माना गया है शुक्र ग्रह का रंग है। काला रंग शनि का है। चित्र चाहे मूर्त हो अथवा अमूर्त, रंग और रेखाओं का समाचीन प्रयोग उसे एक ठोस आधारभूमि देता है।

\section{रागध्यान चित्रावलियों में प्रयुक्त रंग, रेखाएं एवम् प्रतीक}

रंग पहाड़ी वाला की विभिन्न शैलियों में रंगो का वैविध्य देखा जा सकता है अर्थात् कांगड़ा कलम में रंगो का चयन और उपयोग जिस ढ़ग से हुआ है वह बसोहली कलम से भिन्न है। बसोहली कलम में मुख्य व प्राथमिक रंगों (लाल, नीला और पीला) का ही अधिकांशतः प्रयोग हुआ है लेकिन कांगड़ा व गुलेर कलम के चित्रों में इन रंगों के विभिन्न मिश्रण नज़र आते हैं। बसोहली की पृष्ठभूमि का अंकन किसी एक रंग या अधिक रंग की एक ही घसीट में हुआ है। लेकिन काँगड़ा अथवा गुलेर की पृष्ठभूमि में रंगो की विविधता है और साथ ही सूक्ष्मता भी। दोनों शैलियों की अग्रभूमि में भी बड़ा भेद है।

बसोहली कलम में प्राथमिक व मुख्य रंगों के अधिकांश उपयोग से यह सीधा संकेत मिलता है कि यह शैली कांगड़ा कलम की अपेक्षा लोककला के अधिक नज़दीक रही है। बसोहली तथा उस जैसी शैली को छोड़कर जब हम कांगड़ा, बिलासपुर, गुलेर शैलियों में बने चित्रों का देखते हैं तो उनका एक स्पष्ट गुण, उनका सूक्ष्म ओर विस्तारपूर्ण होना नजर आता है। इसका कारण समझना कठिन नहीं। बाहर से विशेषतः मुगल दरबार के चितेरे जब पहाड़ी रियासतों की ओर मुड़े तो सबसे पहले इन्ही रियासतों में बसे। 
यहां वे मुगल राज्य के अन्तर्गत अर्जित विशेषताओं और गुणों को प्रदर्षित करते हैं सूक्ष्मता और विस्तारपूर्ण आलेखन तथा चित्रण विशिष्टत मुगल शैली की ही देन है। बाद में अपने प्रश्रयदाताओं की अभिरूचि के अनुकूल उन्होंने न केवल अपने विषयों को ही चुना बल्कि मुगलकालीन कला की अपेक्षा रंग और रेखाओ के प्रयोग में अधिक दक्षता का परिचय भी दिया।

रेखाएँ

रेखाओं के सम्बंध में भी बसोहली और कांगड़ा शैलियो के चित्रों मे प्रर्याप्त अन्तर देखा जा सकता है। बसोहली शैली की रेखाकृतियाँ कांगड़ा शैली की रेखाकृतियों की तरह सूक्ष्म व सुधड़ नहीं अपितु स्थूल है। कांगड़ा कलम की रेखाएं बसोहली की अपेक्षा अधिक सुन्दर, सूक्ष्म प्रवहमान, लयात्मक, सजीव तथा आकर्षक है। वहाँ हर रेखाकृति हर प्रतिमान से संतुलित है। लेकिन बसोहली की रेखा कृतियों में यह संतुलन नहीं। यहां यदि कलाकार ने आंखे आकर्षक बनाने के लिए उन्हें बड़ा बनाया है। तो वे इतनी बड़ी बन गई है कि सम्पूर्ण आकृति से उनका तालमेल नहीं बैठता। कांगड़ा कलम में नारी की आकृतियां तो सुधड़ रेखाओं से बनी इतनी आकर्षक लगती हैं जैसे उनकी उपमा ढूंढने पर भी नहीं मिले। जहाँ कांगड़ा कला की आकृतियों में रेखाएं प्रवहमान नज़र आती हैं। वहां बसोहली कला में रेखाओं का प्रवाह किन्ही कोणों के समावेश से अवरूद्ध सा हो गया है। यह शैलीगत गुण तो माना जा सकता है लेकिन कांगड़ा कलम की अपेक्षा हीन ही होगा।

यह कोणाकार अंकन केवल किन्हीं आकृतियों के अंगो में ही नहीं, प्रकृति के अंकन में भी दिखाई पड़ता है। वृक्ष तथा पत्तों तक का अंकन नुकीले ढ़ग से हुआ है। कांगड़ा कलम में रेखाओं की यह चुभन नहीं। पहाड़-पहाड़ियों का अंकन हो अथवा वृक्ष-पत्तों का, रेखाएं इन कोणों से बचती हुई प्रवहमान हुई है। यहाँ सम्पूर्ण लयात्मकता प्रवाहपूर्ण है।

\section{पहाड़ी शैलिया में प्रयुक्त प्रतीक एवम् विम्ब}

भारतीय कला में मानवीय और दैविक तत्वों के मध्य जो संबध-व्यवहार नज़र आता है वही कला की आत्मा हैं और वह अपनी अभिव्यक्ति में लक्षण, प्रतीक और बिम्बों का सहारा लेती है। सत्-चित्-आनन्द में सौन्दर्य-सृष्टि की व्याख्या मिलती है। आदि स्पष्टा ने अपने सृजन व निर्माण में सत् कला से द्रव्य लिया, चित् कला से प्राण रस लिया और इस प्रकार आनन्द अवस्था में उसने सौन्दर्य सृष्टि की । ब्रह्मा ने सृष्टि को जन्म दिया था। विष्णु उसका पालक बना और महेश उसका संहारक। सृष्टि के पालक विष्णु की पत्नी शक्ति है जो अपने विशुद्ध सौन्दर्य के प्रतिनिधि के रूप मे लक्ष्मी के नाम से परिचित है। शाक्ति के रूप में जहाँ वह मातृस्थानीय है, लक्ष्मी के रूप में वह सौन्दर्य वैभव को दर्शाती है। विष्णु स्वयं जिन अंलकरणों व उपादनों से विभूषित हैं वे प्रत्यक्षतः ही सार्थकता लिए हुए है। विष्णु की मूर्ति अथवा चित्र में जितना महत्व शंख चक्र गदा तथा पद्य का है उतना उनकी वेशभूषा का नहीं है। यही उपादान उनके व्यक्तित्व को अपनी सम्पूर्णता में प्रस्तुत करते हैं। यहां शंख ऐश्वर्य, चक्र संगठन तथा वीरता और कमल वैराग्य के भाव को परिलक्षित करता है।

\section{प्रकृतिक अंकन}

पार्श्वभूमि में प्रकृति का अंकन भी अत्यन्त सजीव व सार्थक है। प्रकृति की उत्फुल्लता मानवीय व्यवहार के परिप्रेक्ष्य में अत्यन्त मनमोहक लगती है। धूप और वर्षा में जहाँ प्रकृति अपने रंग बदलती है वहां क्रीडापूर्ण भूमिका सहज निखरती जाती है। निकुजों की सघन छाया, शीतल चाँदनी, यमुना तट पर कदम्ब वृक्षों का फलना फूलना, पक्षियों का कलरव और पशुओं की चापल्यपूर्ण क्रीड़ा का वर्णन मिलता है। इसी प्रकार शिव तथा पार्वती संबधी चित्रों के लिए जिन कठोर गगन चुम्बी हिम मण्डित पर्वत श्रृंगों का दूर बैठे कवियों ने वर्णन किया है। सूर्य की पहली किरण से सूर्यास्त तक प्रकृति जिन रंगों से गुजरती है तथा रात्री का सौन्दर्य भी अपने आयामों में खूब खिलता है फिर चाहे यह तारों भरी रात्रि का मूक सौन्दर्य या अथवा बादलों से छितराती चांदनी, वर्षा ऋतु तथा भंवरों का मंडराना आदि इस समस्त सृजन में गहर अनुभूति तथा मनोभावों के अनुरूप ऋतु और वातावरण की लाक्षणिता और मूर्तद रूपायित हुई है। प्रकृति चित्रण में मुद्राओं का अंकन और रंगो का चयन जिस ढंग से हुआ है वह भावाभिव्यक्ति को अत्यन्त समर्थ व सक्ष्म बनाता है। यही प्रतीकात्मकता और बिम्बविधान का अन्तनिर्हित रहस्य भी मुखरित हुआ देखा जा सकता है। 


\section{प्रतीकात्मक अभिव्यक्ति}

पहाड़ी चित्रकला में जिस खूबी से नारी सौन्दर्य को ऑका गया है। उस खूबी से पुरूष का चित्रण नहीं हुआ है। नारी की आकृतियाँ अपने आप में तो आकर्षक हैं ही, प्रतीकों के उपयोग से सोने पर सुहागे का काम हो गया है। वन में जहाँ कहीं केले के पतों का विछावन के रूप में उपयोग हुआ है नारी की मनसिकता के संस्पर्श की यह प्रतीकात्मक अभिव्यक्ति मानी जा सकती है। लता का पेड के इर्द-गिर्द लिपटना भी स्त्री और पुरूष के संसर्ग की ओर संकेत करता है। नायक-नायिका अथवा कृष्ण और राधा को रूपान्वित करने वाले चित्रों में पक्षियों का जोड़ा भी दिखाई देगा। कृष्ण रंग से काला है और राधा गोरी। कृष्ण और राधा की इन चारित्रिक विशेषताओं के समर्थन में समस्त प्रकृति में अत्यंत सामजस्पात्मक ढंग से किन्ही प्रतीकों की सार्थकता पहचानी जा सकती है जैसे दो वृक्षों में एक श्याम और दूसरा गीरवर्ण।

कमल तो भारतीय संस्कृति में सनातन रूप से आरूढ़ है। लक्ष्मी को कमला भी कहा जाता है। पानी मे उत्पन्न कमल पानी से ही सृष्टि की उत्पति का बोधक भी है। पहाड़ी चित्रों में फूलों, विशेषतः कमल का समावेश पाने का आनन्द के रूप में विस्फोट का परिचायक है। प्रेम की अभिव्यक्ति अनेक रूपों में हुई है। प्रेम के रास्तें में ही जब प्रेमिका अथवा प्रेमी रूठ पड़ता है धीरज खो डालता है। तो उसके लिए प्रकृति में आंधी और तूफान आदि से प्रतीकों का सहारा लेकर पहाड़ी चित्रकला की भावभूमि सम्मन्न की गई है।

पहाड़ी राग माला चित्रकला समस्त भारतीय कला के विकास में एक पड़ाव है। इसलिए परम्परानुसार भारतीय कला के आदि प्रतीकों का प्रचलन यहाँ भी बराबर बना रहा। इस प्रतीकों में कमल, हंस और हाथी प्रमुख है। जो अनेक देवी-देवताओं के साथ संयुक्त दिखाई देते हैं। इनके आधार पर हम किसी भी कलाकृति की यह पहचान कर सकते हैं कि वह भारतीय है या अभारतीय। कमल हंस और हाथी इन तीनों ही प्रतीकों का सम्बन्ध जल से है। कमल की उत्पति जल से है। हाथी की सम्पर्क जल और थल से रहता है। हंस जल, थल और आकाश तीनों में विचरण करता है।

\section{वाहन}

भारतीय देवी देवता बाहन के साथ ही दिखाई देते है। हंस आदि वेदज्ञ ब्रह्मा के रूप मे। हाथी सी सामर्थय वाले गणपति का वाहन चूहा है। इसी प्रकार जल और वर्षा के देवता इन्द्र की अनुरूपता भी ऐरावत अथवा हाथी के साथ सहज ही देखी जा सकती है। देवी अथवा दुर्गा का वाहन सिंह है। देवी दुष्टदालनी है और सिंह जैसा जानवर ही दुष्टों के संहार में शक्ति का प्रबल प्रतीक हो सकता है।

\section{पशु पक्षियों के अंकन में प्रतीककात्मक अभिव्यक्ति}

पहाड़ी रागमाला चित्रों में प्रेम ही मुख्य विषय है इसलिए प्रेम के अनुराग और विराग के अनेक गीत गाने वाला पपीहा सामान्यता नजर आता है। पपीहा युगल कृष्ण और राधा के सम्बंध को बखूबी मुखरित करता है। यदि ये आपस में खुश है तो पपीहा युगल भी वैसी ही दिखाई देगें। ऐसी ही प्रतीकात्मक अभिव्यक्ति सारस, युगल, तोता, मैना, चकोर, चकोरी, मोर-मोरनी आदि के अंकन में सरुचि देखी जा सकती है।

\section{पेड़ पौधो के अंकन में प्रतीकात्मक अभिव्यक्ति}

पेड़ और पौधो के अंकन में प्रतीकात्मक अभिव्यक्ति अत्यन्त रूचिकर है। केले को पौधा राधा अथवा नायिका के मासल सौन्दर्य का प्रतीक है जो पहाड़ी चित्रों में सहज ही देखने को मिलेगा। राधा के सौन्दर्य को प्रतिबम्बित करता हुआ कचनार का पौधा है जो गुलाबी फ़लो से लदा हुआ है एक अत्यन्त सुन्दर व सामान्य प्रतीक है इसी प्रकार लता का पेड़ के इर्द-गिर्द लिपटना अथवा वृक्ष का सहारा लेना राधा और कृष्ण के प्रेम को प्रतीकात्मकता रूप से सर्मथ बना डालता है।

मेघो का उमड़ना, बिजली का कड़कना और वर्शा की फुहार पात्रों की भूमिका को अधिक सार्थक बना देती है। भारतीय कला चेतना और भाव-कल्पना इन प्रतीकों में केन्द्रीत है और उन्ही के सहारे उसे आवश्यक उद्बोधन मिलता है। 